\title{
NQDI-1, an inhibitor of ASK1 attenuates acute perinatal hypoxic-ischemic cerebral injury by modulating cell death
}

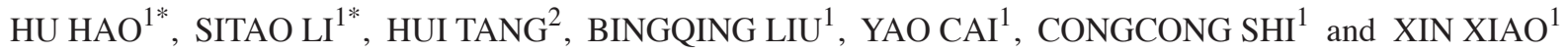 \\ ${ }^{1}$ Department of Pediatrics, The Sixth Affiliated Hospital of Sun Yat-Sen University, Guangzhou, Guangdong 510655; \\ ${ }^{2}$ Central Laboratory, The First Affiliated Hospital of Jinan University, Guangzhou, Guangdong 510630 P.R. China
}

Received May 9, 2015; Accepted March 29, 2016

DOI: $10.3892 / \mathrm{mmr} .2016 .5123$

\begin{abstract}
Apoptosis signal-regulating kinase 1 (ASK1) is a ubiquitously expressed protein kinase, which regulates cell fate in numerous injury conditions. Therefore, ASK1 may be a promising novel therapeutic target for injury. However, the expression and distribution of ASK1 in the perinatal brain following hypoxia-ischemia (HI) remains to be elucidated. In the present study, western blotting and immunofluorescence were used to determine the expression and distribution of ASK1 and any associated downstream targets in the perinatal rat brain following HI. NQDI-1, a specific inhibitor of ASK1 was intracerebroventricularly injected following neonatal rats brain insult for neuroprotection. The results revealed an increased expression of ASK1 and this expression was localized to the neurons and astrocytes, compared with the sham controls. Additionally, it was demonstrated that the ASK1/ c-Jun N-terminal kinases (JNK) pathway was involved in the brain damage following HI in neonatal rats. Notably, NQDI-1 significantly inhibited the in vivo expression levels of ASK1, phosphorylated (p-)JNK, p-c-Jun, p53 and caspase 3. Reduced acute hypoxic-ischemic cerebral injury and cell apoptosis was observed following the injection of NQDI-1. Collectively, NQDI-1 attenuated acute perinatal hypoxic-ischemic cerebral injury by inhibiting the expression of ASK1 and cell apoptosis. This may be a promising novel neuroprotective inhibitor for perinatal cerebra injury.
\end{abstract}

\section{Introduction}

Hypoxia-ischemia (HI) is a major cause of perinatal brain injury and results in mortality or lifelong morbidities $(1,2)$.

Correspondence to: Dr Xin Xiao, Department of Pediatrics, The Sixth Affiliated Hospital of Sun Yat-Sen University, 26 Yuancun Erheng Road, Guangzhou, Guangdong 510655, P.R. China

E-mail: txiaoxin1049@163.com

*Contributed equally

Key words: ASK1, NQDI-1, perinatal hypoxia-ischemia, apoptosis, brain
Hypoxic ischemia (HI) in neonates triggers sequential cascades of neurotoxic events within hours, which last for days to weeks following the injury, resulting in significant neuronal injury $(2,3)$. The incidence of perinatal HI reaches 2-6\% in live term births each year in Western countries $(4,5)$. Approximately half of the HI events result in mortality and $25 \%$ of the survivors suffer from neurological disabilities, including cerebral palsy, cognitive and/or sensory deficits, mental retardation, learning disabilities and epilepsy. These impairments significantly impact life experience and social welfare $(6,7)$. Additionally, the pathophysiological mechanisms are complex and processes including apoptosis, necroptosis, mitochondrial impairment, oxidative stress and inflammation are involved (8). Until now, no effective therapy to treat these neurological disorders exists. Therefore, searching for novel and more effective therapeutic interventions is necessary and of social value.

Apoptosis signal-regulating kinase 1 (ASK1) is one of $>20$ members that make up the triple mitogen-activated protein kinase (MAPK) family of enzymes (9). Over the past decade, numerous previous studies have revealed that ASK1 serves a pivotal role in the cellular response to a wide variety of environmental and biological stresses, including reactive oxygen species such as hydrogen peroxide, endoplasmic reticulum stress caused by protein aggregation, influx of calcium ions, and receptor-mediated signals transduced via lipopolysaccharides (LPS), Fas ligand, cytokines (TNF $\alpha$ ) and certain $G$ protein-coupled receptor agonists (10-14). In addition, exogenous expression of ASK1 in cells has shown that ASK1 signaling engages the intrinsic apoptosis pathway, promoting cytochrome $c$ release from mitochondria and the subsequent activation of caspase 3 and $9(15,16)$.

Further notable evidence indicated that ASK1 serves a potential role in the pathogenesis of ischemic brain injury (17-19). Using a cerebral ischemia rat model, as well as in an in vitro kinase assay, ASK1 exhibited increased auto-phosphorylation and activity at various time points following the induction of cerebral ischemia (20). Heat shock protein-27 (Hsp27) was observed to be upregulated in cells surviving ischemic insults and in ischemic preconditioning models (21). Additionally, Hsp27 promoted long-term neuroprotection against cerebral ischemia by physically interacting with ASK1 resulting in the inhibition of ASK1 activity (22). Genetic knockdown 
of ASK1 or inhibition of the ASK1/MAPK kinase (MKK)4 cascade also effectively abolished neuronal ischemia (22). Therefore, inhibition of the pro-apoptotic ASK1 pathway may be a promising novel neuroprotective strategy for cerebral injury.

However, the expression and distribution of ASK1 in the brain of perinatal HI rat models remain to be elucidated. In the present study, the 7-day-old rat was used to build the HI model. At several time points following the insult, ASK1 expression and the distribution were determined by western blotting and double immunofluorescence, respectively, and indicated that ASK1 was observed and localized within neurons and astrocytes. It was also demonstrated that the ASK1/JNK pathway was involved in the brain damage following HI in neonatal rats. Notably, NQDI-1, a specific inhibitor of ASK1 was intracerebroventricularly injected following insult of the neonatal rat brain and was demonstrated to significantly attenuate acute hypoxic-ischemic cerebral injury by inhibiting cell apoptosis.

\section{Materials and methods}

HI rat model and treatments. All animal procedures were approved by the Sun Yat-Sen University Committee on Animal Use and Care. A total of 12 female Sprague-Dawley rats with litters of mixed gender pups were acquired from the Nanjing University (Nanjing, China). The mothers were housed at $25^{\circ} \mathrm{C}$ under a 12-h light/dark cycle, with ad libitum access to food and water, until the pups were 7-days-old. The HI model was established, as described previously $(23,24)$. Briefly, each pup was anesthetized with diethyl ether $(100 \mathrm{mg} / \mathrm{kg}$; Sigma-Aldrich, St. Louis, MO, USA) during the entire procedure and the body was maintained at $37^{\circ} \mathrm{C}$ using a homoisothermy bench. Following a $0.5 \mathrm{~cm}$ skin incision in the midline of the neck, the right common carotid artery (CCA) was permanently ligated with 5-0 silk. Following ligation of the CCA, the pups were returned to their housing for $0.5 \mathrm{~h}$ to recover from anesthesia. The pups were subsequently maintained in a hypoxic chamber at $37^{\circ} \mathrm{C}, 8 \% \mathrm{O}_{2}$ and $92 \% \mathrm{~N}_{2}$ for $6,12,24$ or $48 \mathrm{~h}$. The sham group underwent a neck dissection and the silk was placed around the CCA, but was not ligated. The pups were anesthetized with $2.5 \%$ halothane and were intracerebroventricularly infused with dimethyl sulfoxide (DMSO; Sigma-Aldrich) or 250 nmol NQDI-1 (Cayman Chemical Company, Inc., Ann. Arbor, MI, USA), a highly specific ASK1 inhibitor, dissolved in DMSO into the right cerebral hemisphere 30 min prior to HI using a 30-gauge needle with a $5 \mu \mathrm{l}$ Hamilton syringe (infusion rate, $1 \mu \mathrm{l} / \mathrm{min})$. The NQDN-1 dose ( $250 \mathrm{nmol} / \mathrm{pup})$ used in the present study was selected, according to a previous report (25).

Immunofluorescence. At 6,12, 24 or $48 \mathrm{~h}$ following HI, the cortices of the pups were harvested and cut into 5-mm sections for further analyses. Immunofluorescence was performed using the 24-h sections only. Sections were embedded with paraffin and incubated with rabbit anti-ASK1 polyclonal antibody (1:200; cat. no. ab131506; Abcam, Cambridge, UK) or rabbit anti-p-JNK monoclonal antibody (Thr183/Tyr185; 1:200; cat. no. 9255; Cell Signaling Technology, Inc., Beverly, MA, USA), and mouse anti-NeuN (1:150; cat. no. MAB377; EMD Millipore, Billerica, MA, USA) or mouse anti-GFAP
(1:80; cat. no. MAB360; EMD Millipore) monoclonal antibodies. Subsequently, the sections were incubated with fluorescein isothiocyanate-conjugated goat anti-rabbit (1:120; cat. no. sc-2012; Santa Cruz Biotechnology, Inc.) or goat anti-mouse (1:120; cat. no. sc-2010; Santa Cruz Biotechnology, Inc.) immunoglobulin $\mathrm{G}(\mathrm{IgG})$, and tetramethylrhodamine-conjugated goat anti-rabbit (1:120; cat. no. sc-2780; Santa Cruz Biotechnology, Inc.) or goat anti-mouse (1:120; cat. no. sc-2781; Santa Cruz Biotechnology, Inc.) IgG. The nucleus was stained with 4',6-diamidino-2-phenylindole (DAPI; Beyotime Institute of Biotechnology, Inc., Dalian, China; 1:100). A total of 5 sections per rat were analyzed under a microscope (DTX500; Nikon Corporation, Tokyo, Japan).

Western blot analysis. Isolated cortices and hippocampi from the right hemisphere were dissected ( $\mathrm{n}=4$ /group). Cortices were homogenized in ice-cold lysis buffer (Beyotime Institute of Biotechnology, Inc.) containing several protease inhibitors, including phenylmethanesulfonyl fluoride $(0.5 \mathrm{mmol} / \mathrm{l}$; Sigma-Aldrich), aprotinin (5 $\mu \mathrm{g} / \mathrm{ml}$; Sigma-Aldrich) and leupeptin ( $5 \mu \mathrm{g} / \mathrm{ml}$; Sigma-Aldrich), and a phosphokinase inhibitor (10 $\mu \mathrm{g} / \mathrm{ml}$; Sigma-Aldrich). The lysates were centrifuged at $13,000 \mathrm{x} \mathrm{g}$ for $30 \mathrm{~min}$ at $4^{\circ} \mathrm{C}$. Protein concentrations were determined using a bicinchoninic acid protein assay kit (Pierce Biotechnology, Inc., Rockford, IL, USA) with bovine serum albumin as the standard. The protein samples (30 $\mu \mathrm{g} / \mathrm{lane})$ were separated on $8-12 \%$ sodium dodecyl sulfate-polyacrylamide gels. The proteins were subsequently transferred onto polyvinylidene fluoride membranes. The membranes were blocked with 5\% non-fat dry milk in Tris-buffered saline containing $0.05 \%$ Tween-20 at room temperature for $1 \mathrm{~h}$ with rotation. The membranes were incubated overnight at $4^{\circ} \mathrm{C}$ with rabbit anti-JNK monoclonal antibody $(1: 1,000$; Cell Signaling Technologies, Inc.), rabbit anti-p-JNK (Thr183/Tyr185) monoclonal antibody $(1: 1,000)$, rabbit anti-ASK1 polyclonal antibody (1:800), rabbit anti-c-Jun polyclonal antibody (1:800; cat. no. 9165; Cell Signaling Technologies, Inc.), rabbit anti-p-c-Jun polyclonal antibody (1:800; cat. no. 3270; Cell Signaling Technologies, Inc.), rabbit anti-p53 polyclonal antibody (1:200; cat. no. ab131442; Abcam) and mouse anti-caspase 3 polyclonal antibody (1:100; cat. no. ab3623; Abcam). A mouse anti-GAPDH polyclonal antibody (1:2,000; cat. no. sc-365062; Santa Cruz Biotechnologies, Inc., Santa Cruz, CA, USA) was used as an internal loading control. The membranes were subsequently incubated with peroxidase-conjugated goat anti-rabbit and anti-mouse immunoglobulin G (1:10,000; cat. nos. ZB2301 and ZB2305, respectively; ZSBIO, Beijing, China) in blocking solution for $1 \mathrm{~h}$ at $37^{\circ} \mathrm{C}$. The signals of the bound antibodies were visualized by enhanced chemiluminescence (EMD Millipore). Image-Pro Plus (version 6.0; Media Cybernetics, Inc., Rockville, MD, USA) was used to quantify the densities of the protein signals on X-ray films following scanning. The protein levels were normalized against the protein levels of the loading control, GAPDH.

\section{2,3,5-triphenyltetrazolium chloride (TTC) staining. As} described previously (26), $48 \mathrm{~h}$ after HI induction, TTC staining was performed to measure the infarct volume $(n=4)$. The animals were perfused with cold saline under deep anesthesia and the brains were quickly removed. The brains were 
subsequently embedded in brain matrix and frozen for 3 min at $-80^{\circ} \mathrm{C}$. Each brain was sliced coronally at $2 \mathrm{~mm}$ intervals with the matrix. A total of 4 sliced sections were subsequently stained with $1 \% \mathrm{TTC}(\mathrm{w} / \mathrm{v})$ at $37^{\circ} \mathrm{C}$ for $12 \mathrm{~min}$ and were subsequently fixed in $4 \%(\mathrm{w} / \mathrm{v})$ formaldehyde in PBS for $24 \mathrm{~h}$ at $4^{\circ} \mathrm{C}$. Finally, the brain slice images were captured under a microscope (DTX500) and the areas of unstained tissue (the infarct areas) were delineated manually using Image Pro Plus (version 6.0) software by an individual in a blinded manner.

Terminal deoxynucleotidyl transferase deoxyuridine triphosphate nick-end labeling (TUNEL) assay. TUNEL assays were performed to assess the apoptosis of cells in the rat brain cortex using the DeadEnd ${ }^{\mathrm{TM}}$ Fluorometric TUNEL system (Promega Corporation, Madison, WI, USA), according to the manufacturer's protocol. Cell nuclei with dark green fluorescent staining, which were defined as TUNEL-positive nuclei, were visualized using a fluorescence microscope (DTX500). The number of TUNEL-positive cells were counted in six randomly selected fields. The cell nuclei were then counter-stained with 4',6-diamidino-2-phenylindole, visualized using a fluorescence microscope (DTX500) and counted.

Statistical analysis. Statistical analysis was performed using SPSS version 15.0 (SPSS, Inc., Chicago, IL, USA). Numerical continuous data were presented as the mean \pm standard deviation and were analyzed using one-way analysis of variance. $\mathrm{P}<0.05$ was considered to indicate a statistically significant difference.

\section{Results}

HI induces the expression and distribution of ASK1. The expression levels of ASK1 were determined by western blotting in the rat HI model. A significant increase in the expression of ASK1 was observed in each hemisphere $6 \mathrm{~h}$ after insult, followed by a $>4$-fold increase in the protein expression of ASK1 in the brain of HI model compared with the sham group at 6,12 and $24 \mathrm{~h}$ (Fig. 1A and B). The expression of ASK1 at $48 \mathrm{~h}$ after insult was increased 2.86-fold compared with the sham group (Fig. 1A and B). To further determine the distribution of ASK1 in the rat cortex following HI, double immunofluorescence was performed using paraffin-embedded sections from sham controls, as well as from control rats at $24 \mathrm{~h}$ after $\mathrm{HI}(\mathrm{n}=5 / \mathrm{group})$. The neuronal-specific marker, NeuN, and the astrocyte-specific marker, glial fibrillary acidic protein (GFAP), was used to indicate the neurons. As shown in Fig. 1C, increased expression of ASK1 was observed and ASK1 was localized in the astrocytes at $24 \mathrm{~h}$ after insult, compared with the sham group. NeuN and ASK1 double immunofluorescence indicated increased expression of ASK1 and that it was localized to the neurons at $24 \mathrm{~h}$ after insult, compared with sham group (Fig. 1D). These results indicated that ASK1 was localized to the astrocytes and neurons following HI in developing brain.

HI increases the expression levels of $p$-JNK, p53,p-c-Jun and caspase 3. A previous study indicated that $\mathrm{p}-\mathrm{JNK}$ is the direct target of ASK1 (27). Therefore, western blotting and immuno- fluorescence were used to determine the expression of p-JNK in the rat cortex following $\mathrm{HI}$ at different time points. As shown in Fig. 2A and B, the expression of p-JNK was significantly increased between 6 and $48 \mathrm{~h}$ after insult, compared with the sham controls. Additionally, no significant change in the expression of JNK was observed at different time points following insult. Furthermore, immunofluorescence also indicated an increased expression of ASK1 in the rat cortex following $\mathrm{HI}$ at 6, 12, 24 and $48 \mathrm{~h}$ (Fig. 2C). Western blotting was used to determine the expression of ASK1-associated downstream targets. As shown in Fig. 2D and E, the expression levels of p-c-Jun, p53 and caspase 3 were significantly upregulated between 6 and $48 \mathrm{~h}$ after insult, compared with the sham controls.

NQDI-1 inhibits the expression of ASK1 and downstream targets in the HI model. NQDI-1 has been approved as a inhibitor of ASK1 and is widely used in vitro and in vivo. In the present study, $250 \mathrm{nmol}$ NQDI-1 in DMSO was intracerebroventricularly injected following brain insult. Western blotting was performed to determine the expression of ASK1 in the sham, HI, DMSO and NQDI-1 groups and indicated that NQDI-1 markedly inhibited the expression of ASK1 in the brain cortex, compared with the HI and DMSO group (Fig. 3A and B). Furthermore, immunofluorescence staining also indicated that the expression of ASK1 was inhibited by NQDI-1 in the brain cortex (Fig. 3C). The expression of downstream targets of ASK1 were also determined in the present study. As shown in Fig. 3D and E, the expression levels of p-JNK, p-c-Jun, p53 and caspase 3 were significantly decreased by NQDI-1, compared with the HI and DMSO groups. Low expression of p-JNK in the brain cortex was also observed by immunofluorescence in the NQDI-1-treated group (Fig. 3F).

NODI-1 inhibits injury and apoptosis in the brain cortex. TTC staining was used to evaluate the brain infract volume in the different groups. As shown in Fig. 4A, injection of NQDI-1 markedly decreased the infract volume of the brain at $48 \mathrm{~h}$ after HI. It is known that apoptosis is the major cause of brain injury following HI. Additionally, ASK1, as an inductor of apoptosis, has been widely researched in tumors. Therefore, in the present study, the apoptosis was determined by TUNEL. An increased number of apoptotic cells were observed in the brain cortex of the HI and DMSO-treated rats, compared with the sham controls (Fig. 4B). Notably, the apoptosis of neuron cells in the brain cortex was significantly inhibited following intracerebroventricularly injection of NQDI-1, compared with the HI and DMSO-treated groups. These results indicated that NQDI-1 markedly inhibited apoptosis in the brain cortex.

\section{Discussion}

Numerous previous studies have shown that ASK1 serves a potential role in the pathogenesis of ischemic brain injury (17-19). However, the expression and distribution of ASK1 in the brain of a perinatal HI rat model remains to be elucidated. In the present study, it was shown for the first time, to the best of our knowledge, that the ASK1/JNK 
$\mathbf{A}$

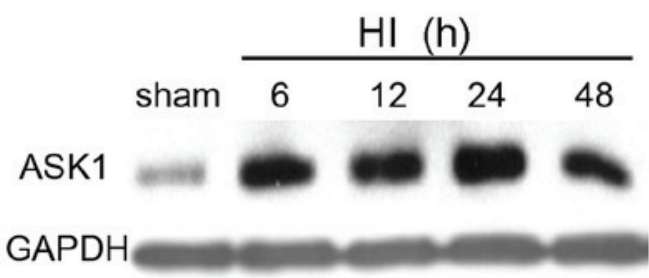

C
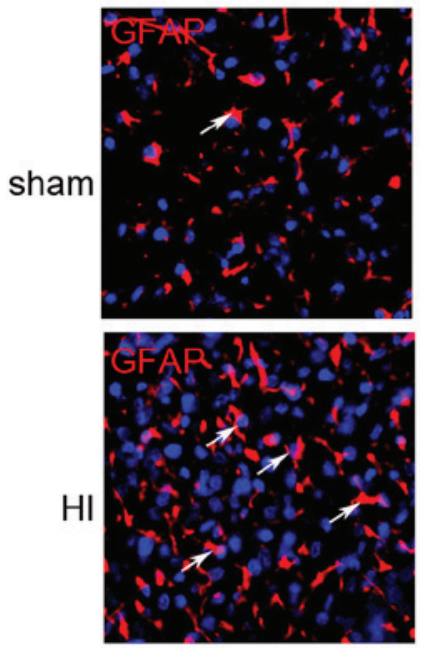

D
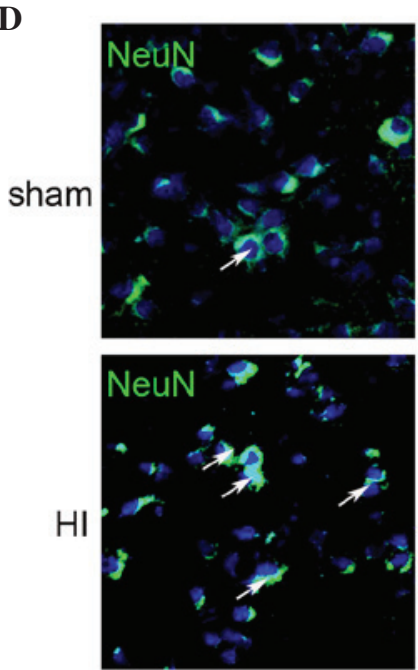
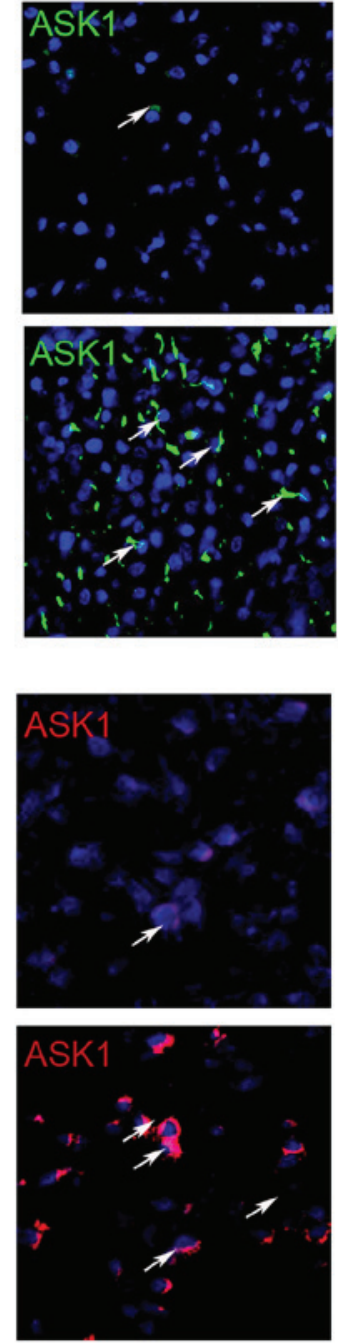

B
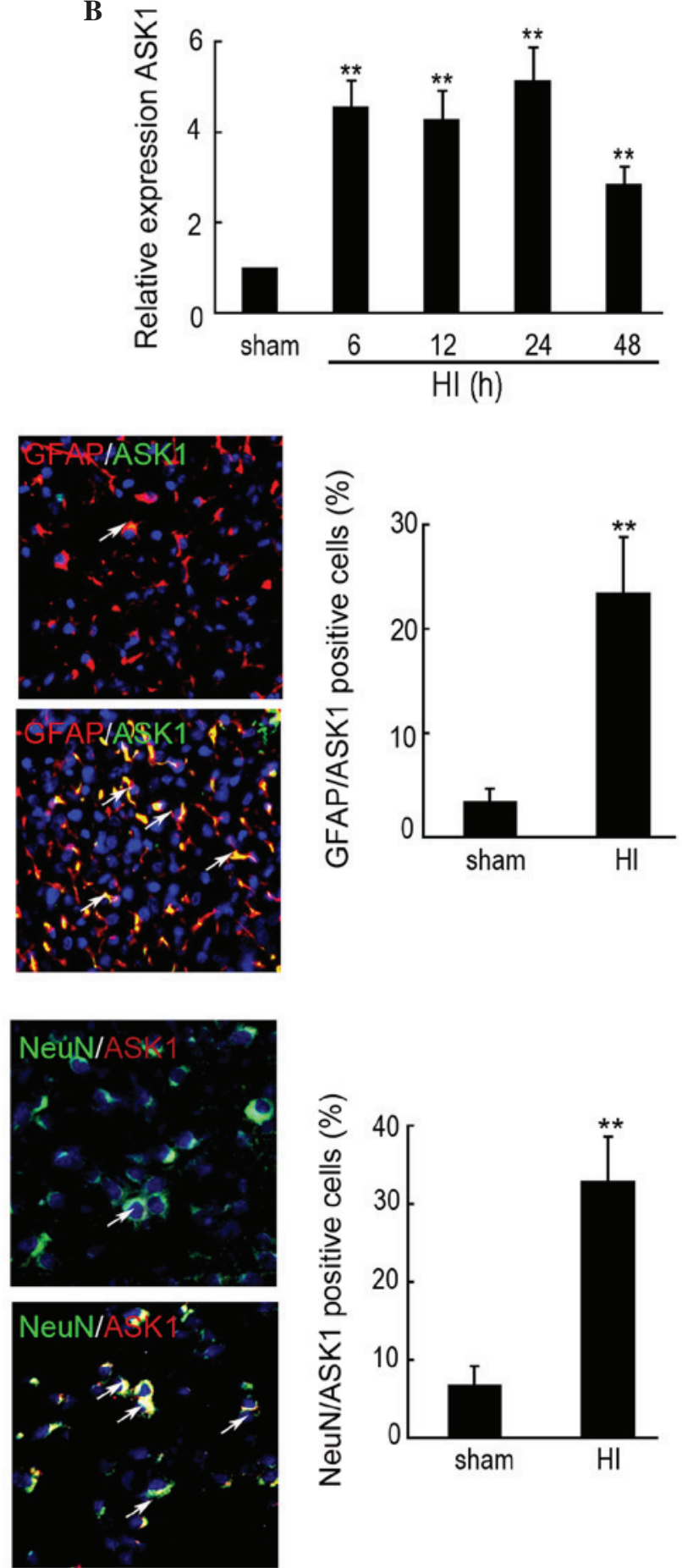

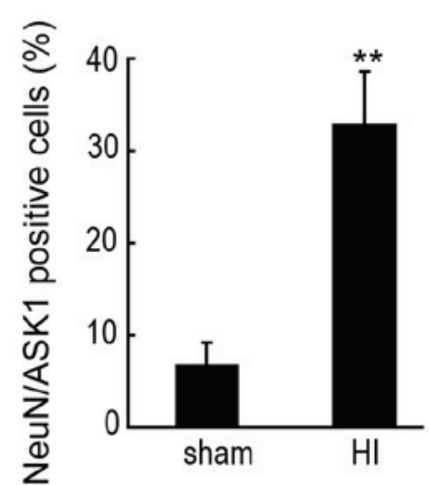

Figure 1. Expression of ASK1 in the brain cortex of the rat HI model was determined by western blotting and immunofluorescence. The brain cortex of the rat was perfused and collected at $6,12,24$ and $48 \mathrm{~h}$ after brain insult. No brain insult was used as a sham control. (A and B) Western blotting detection of the protein expression of ASK1 in the sham and HI model rats at $6,12,24$ and $48 \mathrm{~h}$ after brain insult $\left(\mathrm{n}=4 ;{ }^{* * *} \mathrm{P}<0.01\right)$. GAPDH was used as a loading control. (C) Double immunofluorescence with GFAP (red) and ASK1 (green) was used in paraffin-embedded sections from sham controls, as well as from control rats at $24 \mathrm{~h}$ after $\mathrm{HI}$ ( $\mathrm{n}=6 /$ group; magnification, $\mathrm{x} 200)$. DAPI was used to indicate the cell nucleus. ( $\left.{ }^{* *} \mathrm{P}<0.01\right)$. (D) Double immunofluorescence of NeuN (green) and ASK1 (red) was used in paraffin-embedded sections from sham controls, as well as from control rats at $24 \mathrm{~h}$ after HI ( $\mathrm{n}=6 /$ group). DAPI was used to indicate the cell nucleus. ( ${ }^{* *} \mathrm{P}<0.01$; magnification, $\mathrm{x} 200$ ). ASK, apoptosis signal-regulating kinase 1; HI, hypoxia-ischemia; DAPI, 4',6-diamidino-2-phenylindole; GFAP, glial fibrillary acidic protein.

pathway is involved in neuronal apoptosis in developing rat brain following HI. Additionally, the present study indicated that intracerebroventricularly injection of NQDI-1, a specific inhibitor of ASK1, after neonatal rats brain HI significantly attenuated acute HI cerebral injury by inhibiting cell apoptosis.
ASK1 is a ubiquitously expressed kinase protein, which regulates cell fate in numerous injury conditions and disease models $(17,28,29)$. In the ischemic perfused heart, the activation of ASK1 was observed to have a similar time course compared with that of MKK6 (28). In ischemic brain injury, slight upregulation of ASK1 by LPS promoted the differentiation 
A

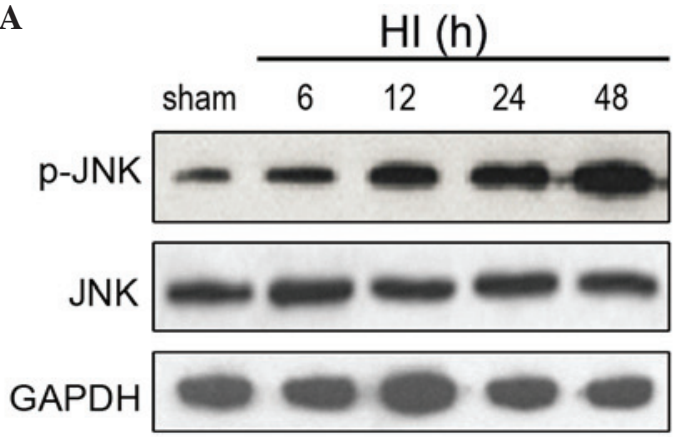

B

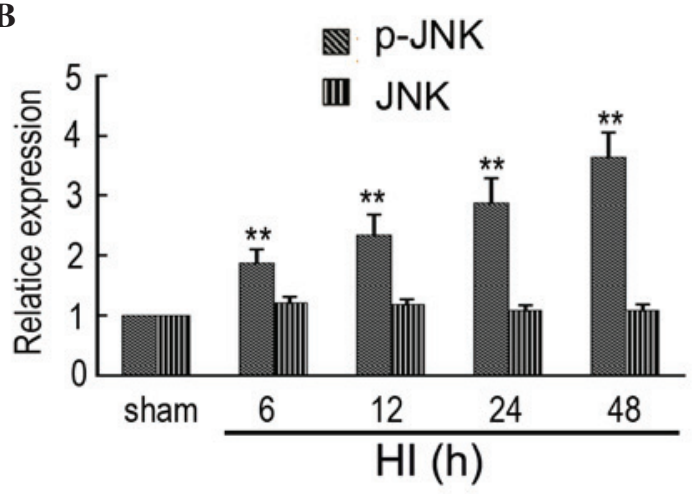

C

D
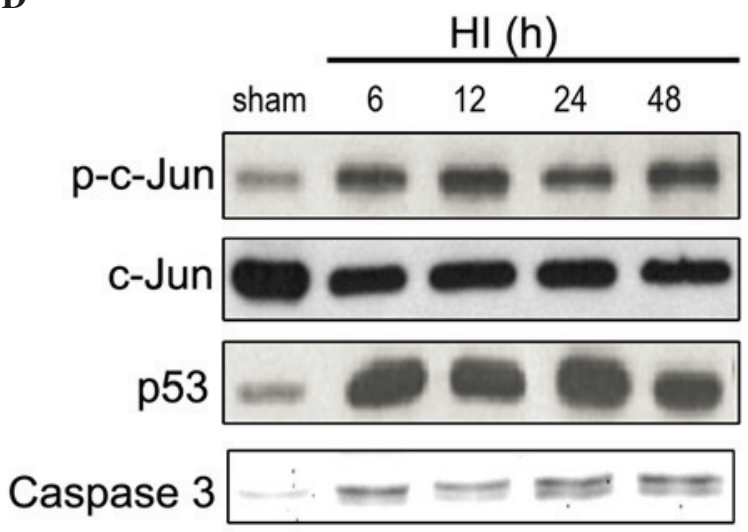

GAPDH sham

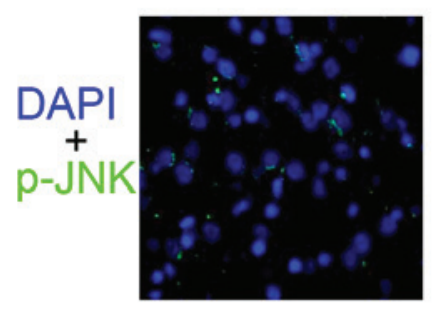

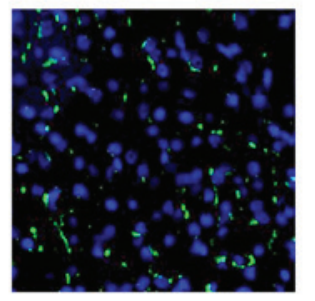

$\mathrm{HI}(\mathrm{h})$
24

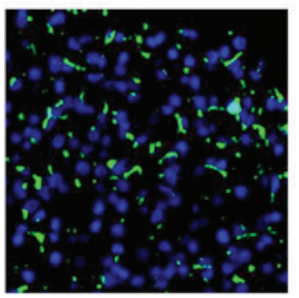

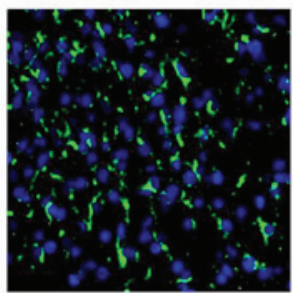

48

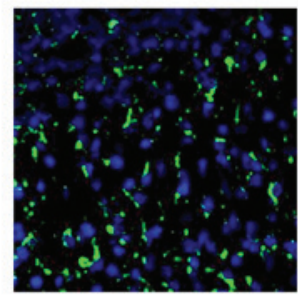

$\mathbf{E}$

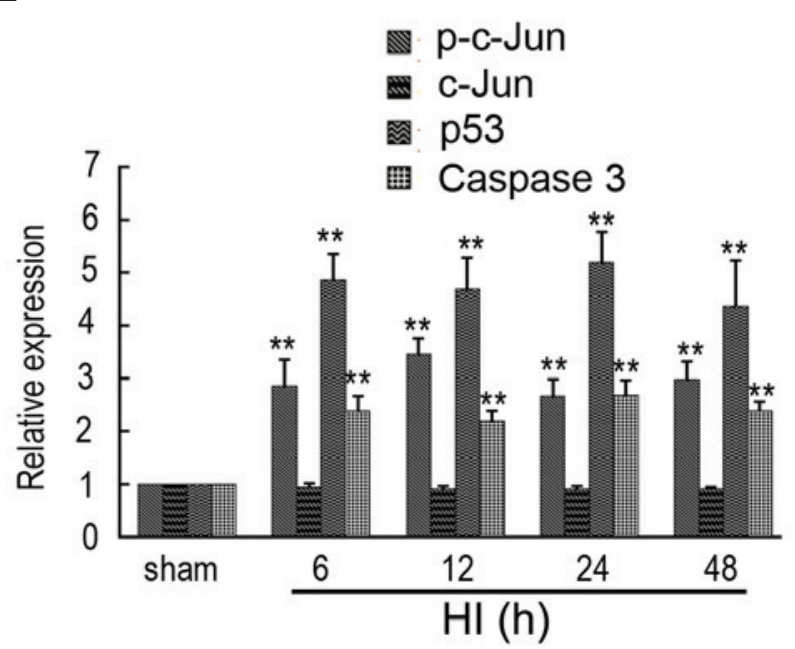

Figure 2. Expression of ASK1-associated downstream targets in the brain of the rat HI model. (A and B) Western blotting detection of p-JNK and JNK expression in the sham and $\mathrm{HI}$ model at $6,12,24$ and $48 \mathrm{~h}$ after brain insult. GAPDH was used as a loading control (n=4; $\left.{ }^{* *} \mathrm{P}<0.01\right)$. (C) Immunofluorescence detection of p-JNK in the brain of the sham and HI model at 6, 12, 24 and $48 \mathrm{~h}$ after brain insult. DAPI was used to indicate the cell nucleus (magnification, x200). (D and E) Western blotting detection of p-c-Jun, c-Jun, p53 and Caspase 3 in the sham and HI model at 6, 12, 24 and 48 h after brain insult. GAPDH was used as a loading control $\left(\mathrm{n}=4 ;{ }^{* *} \mathrm{P}<0.01\right)$. ASK, apoptosis signal-regulating kinase 1; HI, hypoxia-ischemia; DAPI, 4',6-diamidino-2-phenylindole; GFAP, glial fibrillary acidic protein.

of endogenous neuronal stem cells into the neurons, however, highly increased ASK1 levels following cerebral ischemic damage led to high levels of cell death (17). The expression and distribution of ASK1 following HI in the brain of the perinatal HI rat model remains to be elucidated. To determine this, 7-day-old rat pups were insulted to establish the HI model, and, at 6, 12, 24, 48 and $72 \mathrm{~h}$ after insult, ASK1 expression and distribution was determined by western blotting and double immunofluorescence. These results indicated that ASK1 was significantly increased at $6 \mathrm{~h}$ after brain insult. It was also demonstrated that ASK1 localized with neurons and astrocytes. These results clarified the expression and distribution of ASK1 in the brain of perinatal HI rat models.

It was previously reported that ASK1 is a serine/threonine protein kinase, which can activate both the SEK1-JNK and MKK3/6-p38 MAPK pathways and constitute a pivotal signaling pathway in cytokine- and stress-induced apoptosis (27). Additionally, JNK activation was observed in 
$\mathbf{A}$

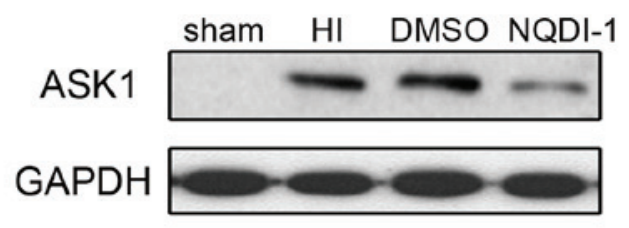

C

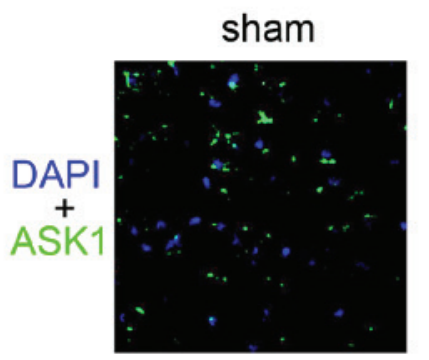

$\mathrm{HI}$

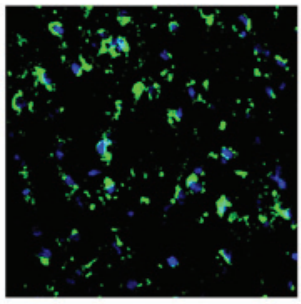

B

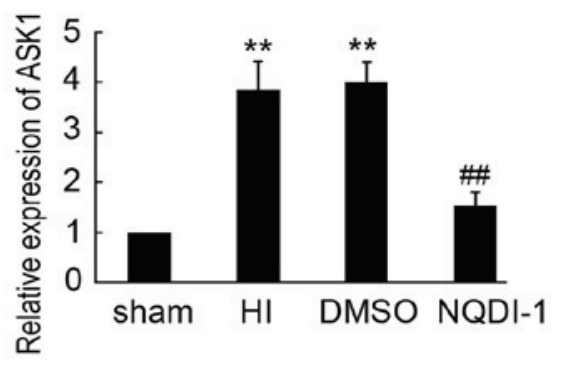

D

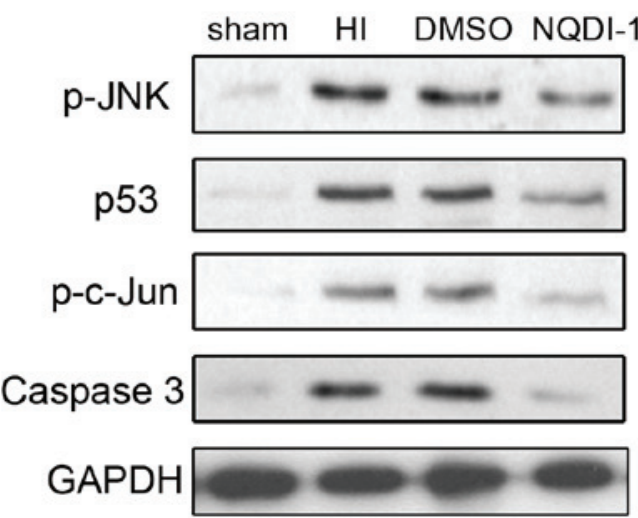

$\mathbf{E}$

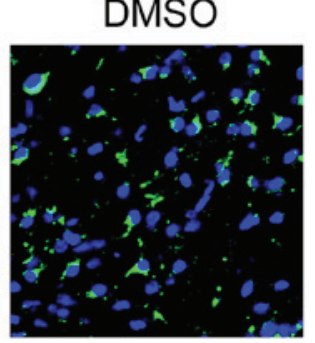

NQDI-1

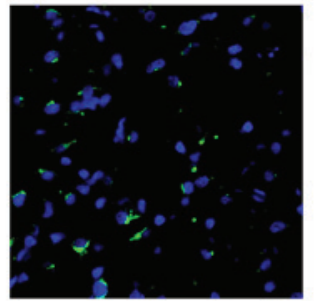

$\mathbf{F}$

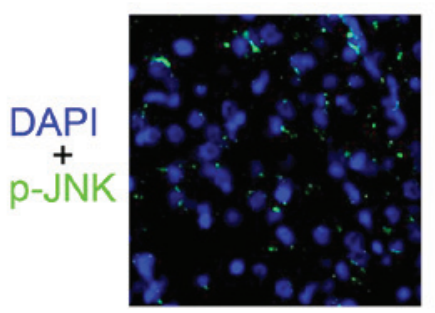

$\mathrm{HI}$

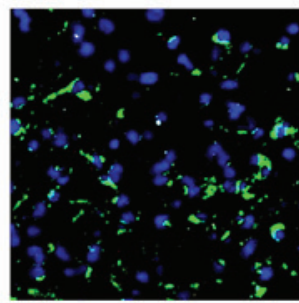

DMSO

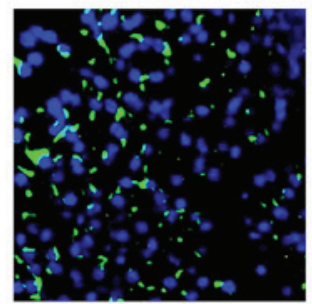

NQDI-1

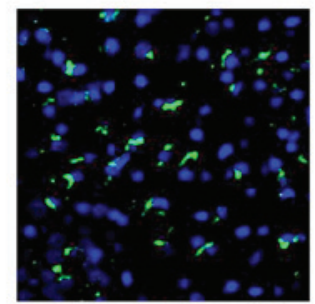

Figure 3. Expression of ASK1 and downstream targets following NQDI-1 treatment in vivo. After 250 nmol NQDI-1 treatment for 48 h, the brain cortex was perfused and collected. (A and B) Western blotting detection of ASK1 expression in the sham, HI, DMSO- and NQDI-1-treated brain cortex ( $\mathrm{n}=4$; ${ }^{* *} \mathrm{P}<0.01$ compared with the sham controls; ${ }^{\#} \mathrm{P}<0.01$ compared with the HI group). (C) Immunofluorescence detection of ASK1 in the sham, HI, DMSO- and NQDI-1-treated brain cortex ( $\mathrm{n}=3$; magnification, $\mathrm{x} 200)$. DAPI was used to indicate the cell nucleus. (D and E) Western blotting detection of p-JNK, $\mathrm{p}$-c-Jun, p53 and Caspase 3 expression in sham, HI, DMSO- and NQDI-1-treated brain cortex $\left(\mathrm{n}=4 ;{ }^{* *} \mathrm{P}<0.01\right.$ compared with the sham controls; ${ }^{\# \#} \mathrm{P}<0.01$ compared with the HI group). (F) Immunofluorescence detection of p-JNK in sham, HI, DMSO-and NQDI-1-treated brain cortex (n=3; magnification, $\mathrm{x} 200$ ). DAPI was used to indicate the cell nucleus. p-, phosphorylated; ASK, apoptosis signal-regulating kinase 1; HI, hypoxia-ischemia; DMSO, dimethyl sulfoxide; DAPI, 4',6-diamidino-2-phenylindole; JNK, c-Jun N-terminal kinase.

several injury diseases, accompanied with changes in the expression of downstream targets, including p-c-Jun, p53, Bim and caspase 3 upregulation and B-cell lymphoma (Bcl)-2 downregulation $(30,31)$. In the present study, the expression of $\mathrm{p}-\mathrm{JNK}$ was determined by western blotting and immunofluorescence. This showed that the expression levels of p-JNK and downstream targets were increased in developing rat brain following HI. These data indicated that the ASK1/JNK pathway is involved in HI-induced cell apoptosis in the perinatal rat brain.

ASK1 is widely used as a therapeutic target in injury diseases. Following ischemia/reperfusion (I/R) in mice, 
A
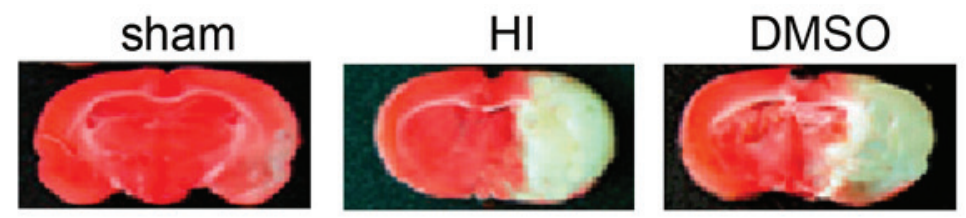

NQDI-1

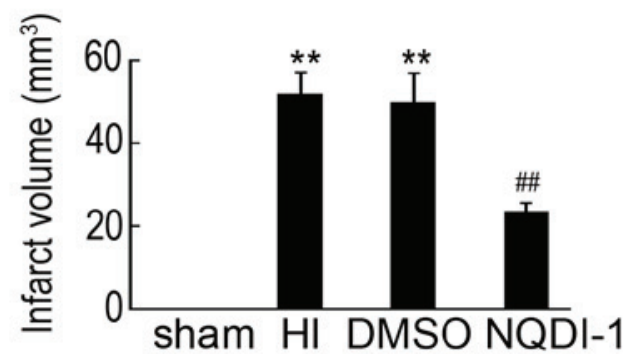

B
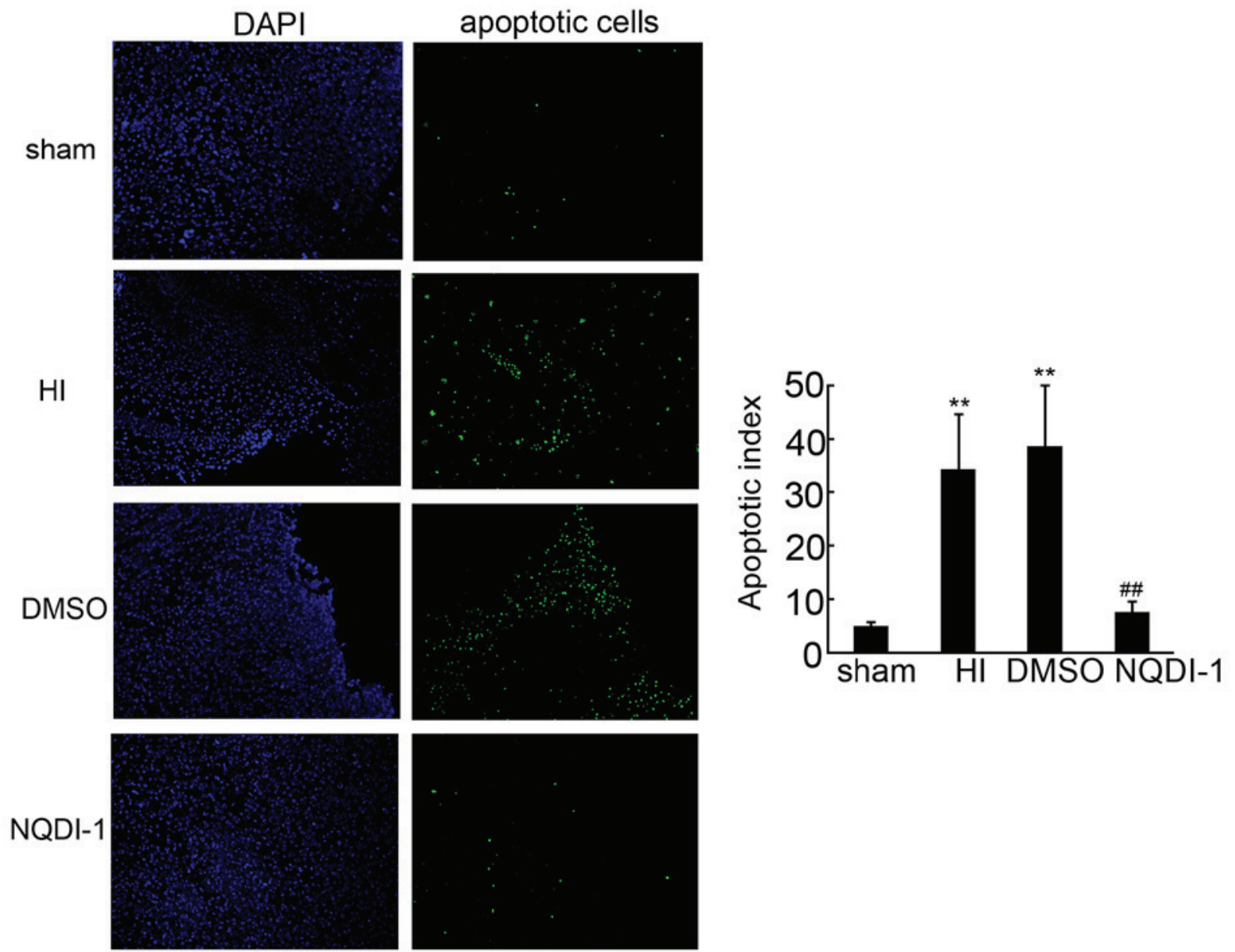

Figure 4. Apoptosis detection by TUNEL in the brain cortex. (A) Infarct volume in the neonatal brain induced by HI was assessed by TTC staining $\left(\mathrm{n}=4\right.$; ${ }^{* *} \mathrm{P}<0.01$ compared with the sham controls; ${ }^{\# \#} \mathrm{P}<0.01$ compared with the HI group). (B) Paraffin-embedded sections from the sham, HI, DMSO- and NQDI-1-treated brain cortex was used for apoptosis detection by TUNEL. DAPI was used to indicate the cell nucleus. Apoptotic cells in each frame were counted and used for apoptosis index analysis $\left(\mathrm{n}=5 ;{ }^{* *} \mathrm{P}<0.01\right.$ compared with the sham controls; ${ }^{\# \#} \mathrm{P}<0.01$ compared with the HI group; magnification, $\left.\mathrm{x} 100\right)$. TUNEL, terminal deoxynucleotidyl transferase dUTP nick end labeling; TTC; 2,3,5-triphenyltetrazolium chloride; HI, hypoxia-ischemia; DMSO, dimethyl sulfoxide; DAPI, 4',6-diamidino-2-phenylindole.

treatment with ASK1-small interfering RNA significantly attenuates the upregulation of ASK1, which was followed by the reduction of infarction in the ischemic brain (18). Administration of NQDI-1 attenuated renal dysfunction and histological changes characteristic of renal I/R injury (IRI), accompanied by the upregulation of superoxide dismutase and $\mathrm{Bcl}-2$ in the kidney (25). Based on the upregulation of ASK1 in the perinatal rat brain following HI, NQDI-1 was intracere- 
broventricularly injected to determine whether inhibition of ASK1 prevents apoptotic neuronal cell death and brain damage following $\mathrm{HI}$ in the perinatal rat. The results indicated that injection of NQDI-1 markedly inhibited the expression of ASK1 following HI in the perinatal rat, accompanied by a decrease of p-JNK, p-c-Jun, p53 and caspase 3, which were the downstream targets of ASK1. TTC staining and the TUNEL assay demonstrated that inhibition of ASK1 by NQDI-1 markedly suppressed infract volume of the brain, according to apoptosis inhibition.

In conclusion, the present study showed for the first time, to the best of our knowledge, that the ASK1/JNK pathway is involved in neuronal apoptosis in developing rat brain following HI. Additionally, the present study indicated that intracerebroventricularly injection of NQDI-1, a specific inhibitor of ASK1, following neonatal rats brain $\mathrm{HI}$ significantly attenuated acute HI cerebral injury by inhibiting cell apoptosis.

\section{References}

1. du Plessis AJ and Volpe JJ: Perinatal brain injury in the preterm and term newborn. Curr Opin Neurol 15: 151-157, 2002.

2. Ferriero DM: Neonatal brain injury. N Engl J Med 351: 1985-1995, 2004.

3. Johnston MV, Fatemi A, Wilson MA and Northington F: Treatment advances in neonatal neuroprotection and neurointensive care. Lancet Neurol 10: 372-382, 2011.

4. Selway LD: State of the science: Hypoxic ischemic encephalopathy and hypothermic intervention for neonates. Adv Neonatal Care 10: 60-66; quiz 67-68, 2010.

5. Wachtel EV and Hendricks-Munoz KD: Current management of the infant who presents with neonatal encephalopathy. Curr Probl Pediatr Adolesc Health Care 41: 132-153, 2011.

6. Bass JL, Corwin M, Gozal D, Moore C, Nishida H, Parker S, Schonwald A, Wilker RE, Stehle S and Kinane TB: The effect of chronic or intermittent hypoxia on cognition in childhood: A review of the evidence. Pediatrics 114: 805-816, 2004.

7. Nelson KB and Lynch JK: Stroke in newborn infants. Lancet Neurol 3: 150-158, 2004.

8. Thornton C, Rousset CI, Kichev A, Miyakuni Y, Vontell R, Baburamani AA, Fleiss B, Gressens P and Hagberg H: Molecular mechanisms of neonatal brain injury. Neurol Res Int 2012: 506320, 2012.

9. Adekeye A, Haeri M, Solessio E and Knox BE: Ablation of the proapoptotic genes CHOP or Ask1 does not prevent or delay loss of visual function in a $\mathrm{P} 23 \mathrm{H}$ transgenic mouse model of retinitis pigmentosa. PloS One 9: e83871, 2014.

10. Ichijo H, Nishida E, Irie K, ten Dijke P, Saitoh M, Moriguchi T, Takagi M, Matsumoto K, Miyazono K and Gotoh Y: Induction of apoptosis by ASK1, a mammalian MAPKKK that activates SAPK/JNK and p38 signaling pathways. Science 275: 90-94, 1997.

11. Chen Z, Seimiya H, Naito M, Mashima T, Kizaki A, Dan S, Imaizumi M, Ichijo H, Miyazono K and Tsuruo T: ASK1 mediates apoptotic cell death induced by genotoxic stress. Oncogene 18: 173-180, 1999.

12. McDonald PH, Chow CW, Miller WE, Laporte SA, Field ME, Lin FT, Davis RJ and Lefkowitz RJ: Beta-arrestin 2: A receptor-regulated MAPK scaffold for the activation of JNK3. Science 290: 1574-1577, 2000

13. Matsuzawa A, Nishitoh H, Tobiume K, Takeda $\mathrm{K}$ and Ichijo $\mathrm{H}$ : Physiological roles of ASK1-mediated signal transduction in oxidative stress- and endoplasmic reticulum stress-induced apoptosis: Advanced findings from ASK1 knockout mice. Antioxid Redox Signal 4: 415-425, 2002.
14. Matsukawa J, Matsuzawa A, Takeda $\mathrm{K}$ and Ichijo $\mathrm{H}$ : The ASK1-MAP kinase cascades in mammalian stress response. J Biochem 136: 261-265, 2004.

15. Hatai T, Matsuzawa A, Inoshita S, Mochida Y, Kuroda T, Sakamaki K, Kuida K, Yonehara S, Ichijo H and Takeda K: Execution of apoptosis signal-regulating kinase 1 (ASK1)-induced apoptosis by the mitochondria-dependent caspase activation. J Biol Chem 275: 26576-26581, 2000.

16. Hao W, Takano T, Guillemette J, Papillon J, Ren G and Cybulsky AV: Induction of apoptosis by the Ste20-like kinase SLK, a germinal center kinase that activates apoptosis signal-regulating kinase and p38. J Biol Chem 281: 3075-3084, 2006.

17. Song J, Cho KJ, Cheon SY, Kim SH, Park KA, Lee WT and Lee JE: Apoptosis signal-regulating kinase 1 (ASK1) is linked to neural stem cell differentiation after ischemic brain injury. Exp Mol Med 45: e69, 2013

18. Kim HW, Cho KJ, Lee SK and Kim GW: Apoptosis signal-regulating kinase 1 (Ask1) targeted small interfering RNA on ischemic neuronal cell death. Brain Res 1412: 73-78, 2011.

19. Wu DN, Pei DS, Wang Q and Zhang GY: Down-regulation of PTEN by sodium orthovanadate inhibits ASK1 activation via PI3-K/Akt during cerebral ischemia in rat hippocampus. Neurosci Lett 404: 98-102, 2006.

20. Zhang Q and Zhang G: Activation and autophosphorylation of apoptosis signal-regulating kinase 1 (ASK1) following cerebral ischemia in rat hippocampus. Neurosci Lett 329: 232-236, 2002.

21. Currie RW, Ellison JA, White RF, Feuerstein GZ, Wang X and Barone FC: Benign focal ischemic preconditioning induces neuronal Hsp70 and prolonged astrogliosis with expression of Hsp27. Brain Res 863: 169-181, 2000.

22. Stetler RA, Cao G, Gao Y, Zhang F, Wang S, Weng Z, Vosler P, Zhang L, Signore A, Graham SH and Chen J: Hsp27 protects against ischemic brain injury via attenuation of a novel stress-response cascade upstream of mitochondrial cell death signaling. J Neurosci 28: 13038-13055, 2008.

23. Kichev A, Rousset CI, Baburamani AA, Levison SW, Wood TL, Gressens P, Thornton C and Hagberg H: Tumor necrosis factor-related apoptosis-inducing ligand (TRAIL) signaling and cell death in the immature central nervous system after hypoxia-ischemia and inflammation. J Biol Chem 289: 9430-9439, 2014.

24. Li D, Qu Y, Mao M, Zhang X, Li J, Ferriero D and Mu D: Involvement of the PTEN-AKT-FOXO3a pathway in neuronal apoptosis in developing rat brain after hypoxia-ischemia. J Cereb Blood Flow Metab 29: 1903-1913, 2009

25. El Eter E: NQDI 1, an inhibitor of ASK1 attenuates acute ischemic renal injury by modulating oxidative stress and cell death. Cardiovasc Hematol Agents Med Chem 11: 179-186, 2013.

26. Tian SF, Yang HH, Xiao DP, Huang YJ, He GY, Ma HR, Xia F and Shi XC: Mechanisms of neuroprotection from hypoxia-ischemia (HI) brain injury by up-regulation of cytoglobin (CYGB) in a neonatal rat model. J Biol Chem 288: 15988-16003, 2013.

27. Matsuzawa A and Ichijo H: Molecular mechanisms of the decision between life and death: Regulation of apoptosis by apoptosis signal-regulating kinase 1. J Biochem 130: 1-8, 2001.

28. Harding SJ, Browne GJ, Miller BW, Prigent SA and Dickens M: Activation of ASK1, downstream MAPKK and MAPK isoforms during cardiac ischaemia. Biochim Biophys Acta 1802: 733-740, 2010.

29. Nako H, Kataoka K, Koibuchi N, Dong YF, Toyama K, Yamamoto E, Yasuda O, Ichijo $\mathrm{H}$, Ogawa $\mathrm{H}$ and Kim-Mitsuyama S: Novel mechanism of angiotensin II-induced cardiac injury in hypertensive rats: The critical role of ASK1 and VEGF. Hypertens Res 35: 194-200, 2012.

30. Zhu Y, Mao XO, Sun Y, Xia Z and Greenberg DA: p38 Mitogen-activated protein kinase mediates hypoxic regulation of Mdm2 and p53 in neurons. J Biol Chem 277: 22909-22914, 2002

31. Liu G, Zhao J, Chang Z and Guo G: CaMKII activates ASK1 to induce apoptosis of spinal astrocytes under oxygen-glucose deprivation. Cell Mol Neurobiol 33: 543-549, 2013. 